\title{
Factors Associated With Fibrate-Induced Creatinine Elevation: Observations in an Outpatient Setting
}

\author{
Ahmed Abbas ${ }^{\mathrm{a}}$, Sanjay Saraf ${ }^{\mathrm{a}}$, Shanath Ramachandran ${ }^{\mathrm{b}, \mathrm{d}}$, \\ Jessie Raju ${ }^{c}$, Sudarshan Ramachandran ${ }^{c}$
}

\begin{abstract}
Background: A reversible rise in creatinine with fibrates has been previously documented. However, this phenomenon remains poorly understood with no associated factors described. We wished to study changes seen in creatinine following fibrate therapy in a routine outpatient setting and identify related risk factors.
\end{abstract}

Methods: Data was collected from the records of patients started on fibrates $(n=132)$ in the lipid clinic at Good Hope Hospital, Sutton Coldfield between 2002 and 2008. Pre and post-fibrate creatinine concentrations were obtained from the pathology database. Creatinine was measured using the Jaffe method on the Roche modular P Unit.

Results: Fenofibrate was used in 117 (88.6\%) patients while the remaining 15 patients were on bezafibrate. Creatinine increased from a mean of $81.9(\mathrm{sd}=17.3$, median $=83$, range: $33-127) \mathrm{umol} / \mathrm{L}$ to $93.8(\mathrm{sd}=20.4$, median $=91$, range: $52-143)$ umol/L following fibrate treatment. Regression analyses showed that male gender (coefficient $=6.64(95 \%$ CI: 0.99/12.29), $\mathrm{P}=0.022)$, pre-treatment creatinine concentration (coefficient $=-0.20$ (95\% CI: $-0.35 /-0.046), \mathrm{P}$ $=0.011$ ), diabetes (coefficient $=-6.37$ (95\% CI: $-12.36 /-0.38), \mathrm{P}=$ 0.037 ) and change in TG (coefficient: $-1.02,95 \% \mathrm{CI}-1.39 /-0.65, \mathrm{P}$ $\leq 0.001$ ) were significantly associated with creatinine change. All the above remained significant when entered into a multiple regression model suggesting independence.

Manuscript accepted for publication June 11, 2012

${ }^{a}$ Department of Medicine, Good Hope Hospital, Heart of England Foundation Trust, Sutton Coldfield, UK

${ }^{\mathrm{b}}$ Department of Clinical Biochemistry, Good Hope Hospital Solihull School, Solihull, UK

${ }^{c}$ Department of Clinical Biochemistry Good Hope Hospital, Heart of England Foundation Trust, Sutton Coldfield, UK

${ }^{\mathrm{d}}$ Corresponding author: Shanath Ramachandran, Department of Clinical Biochemistry, Good Hope Hospital, Heart of England Foundation Trust, Rectory Road, Sutton Coldfield, West Midlands, B75 7RR, UK.

Email: sud.ramachandran@heartofengland.nhs.uk

doi:10.4021/wjnu27w
Conclusions: This study confirms that creatinine increased in our group of patients when fibrates were commenced. Further studies are required to confirm and further explore our findings.

Keywords: Fibrates; Creatinine; Triglycerides; PPAR $\alpha$; Diabetes mellitus

\section{Introduction}

Fibrates are lipid-lowering agents primarily used to reduce raised TG levels. They act by binding and activating the PPAR $\alpha$ receptor, this leading to the activation of a nuclear transcription factor that in turn regulates expression of genes involved in fatty acid and lipoprotein metabolism [1-3]. This results in a modest decrease in LDL-C concentrations, a greater reduction in TG levels and a variable increase in HDL-C concentrations [4].

Creatinine, an amino acid derivative of phosphocreatine and creatine, is an end product of muscle metabolism. About $2 \%$ of creatine is converted to creatinine daily at a fairly constant rate. It is freely filtered through the glomerulus and between $10 \%$ and $40 \%$ (depending on underlying renal function) is secreted by the proximal tubules [5].

A reversible rise in serum creatinine values is included amongst the adverse-effect profile of fibrates. The recent ACCORD-LIPID study demonstrated that serum creatinine levels increased significantly following fenofibrate treatment [6]. The mean increase was from $82 \mu \mathrm{mol} / \mathrm{L}$ to $97 \mu \mathrm{mol} / \mathrm{L}$ at the end of the first year. Similarly, a subgroup analysis of the FIELD study demonstrated a significant increase in mean creatinine levels from $73 \mu \mathrm{mol} / \mathrm{L}$ to $87 \mu \mathrm{mol} / \mathrm{L}$ following five years of fenofibrate treatment [7]. There has been speculation as to the underlying reason for this observation. Proposed explanations have included analytical interference of fibrates with creatinine, increased metabolic production or reduced renal clearance of creatinine.

Hottelart et al in 1999 described a significant $16 \%$ increase in creatinine in 13 dyslipidaemic patients following 2 weeks of fenofibrate treatment [8]. They and Ansquer et al 


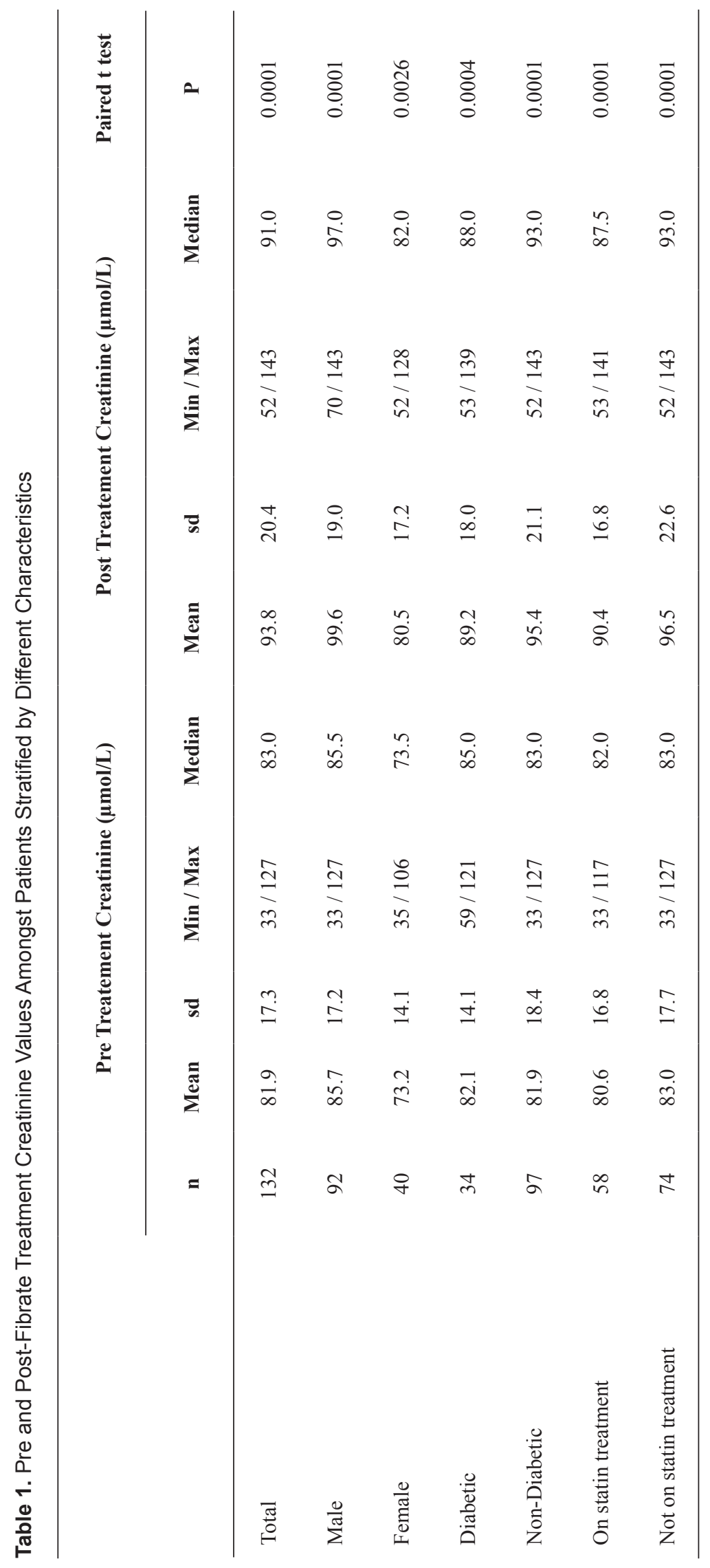


Table 2. Model 1: Association Between Change in Creatinine Concentration (Dependent Variable) and Baseline Factors (Independent Variable) Studied Using Separate Linear Regression Analyses; Model 2: Multiple Regression Analysis With Change in Creatinine (Dependent Variable) and Changes in Lipid Values (Independent Variables). Analysis Corrected for Duration of Fibrate Treatement

\begin{tabular}{|c|c|c|c|c|c|}
\hline & Factor & $\mathbf{N}$ & coefficient & 95\% Confidence Interval & $\mathbf{P}$ \\
\hline \multicolumn{6}{|l|}{ Model 1} \\
\hline & Male & 132 & 6.64 & $1.0 / 12.3$ & 0.022 \\
\hline & Pre treatement Creatinine & 132 & -0.20 & $-0.3 /-0.1$ & 0.011 \\
\hline & Age & 129 & 0.04 & $-0.3 / 0.2$ & 0.782 \\
\hline & Diabetes & 131 & -6.37 & $-12.4 /-0.4$ & 0.037 \\
\hline & Duration of treatment & 124 & 1.70 & $-6.5 / 3.1$ & 0.482 \\
\hline & Statin treatment & 132 & -3.78 & $-9.1 / 1.5$ & 0.16 \\
\hline \multicolumn{6}{|l|}{ Model 2} \\
\hline & Change in TC levels & 109 & 0.54 & $-1.68 / 2.77$ & 0.63 \\
\hline & Change in TG levels & 109 & -1.37 & $-2.43 /-0.32$ & -0.011 \\
\hline & Change in HDL-C levels & 109 & -6.53 & $-6.30 / 19.36$ & 0.32 \\
\hline
\end{tabular}

[9] investigated whether the hypercreatininemia was due to an analytical interference by comparing the Jaffe colorimetric method with HPLC and mass spectrometry. Both studies showed that elevation of creatinine was real and not method dependant. Hottelart et al [10] in a subsequent study and Ansquer et al [9] concluded that an increased metabolic production rate was the most likely cause of the raised creatinine observed as urinary levels of creatinine were not reduced and GFR measured by inulin was not impaired. Davidson et al. also agreed in a review of the safety profile of fibrate therapy that the increase in creatinine observed did not represent a true deterioration in renal function [11]. They noted that on review of various clinical trials describing increased serum creatinine following fibrate therapy, creatinine clearance was not decreased. Interestingly, even as urea, cystatin $\mathrm{C}$ and homocysteine increased in line with the creatinine these studies did not show an expected reduction in GFR.

However, Lipscombe et al [12] suggested that the most likely explanation for the observed phenomenon was an alteration in renal haemodynamics induced by fibrates. They demonstrated a reversible increase in creatinine following fibrate therapy in a group of 10 men, 6 of whom had received a renal transplant with 5 patients on cyclosporine treatment. Similarly Tsimihodimos et al have also proposed that fibrates, probably through their action on PPAR $\alpha$, affect the synthesis of vasodilatory prostaglandins in the kidney [13].
Thus although this phenomenon has been well documented in large randomised controlled trials as well as small patient series, the underlying mechanism remains poorly understood. Patient characteristics associated with it have also not been reported in the literature. It has been our policy to measure serum creatinine in patients attending our lipid clinic at Good Hope Hospital in line with NKD and NLA recommendations before initiating fibrate treatment and on subsequent visits $[11,14]$. In our study, we wished to confirm this expected increase in creatinine and also determine any baseline factors that may be associated with any change in creatinine levels.

\section{Subjects and Methods}

Patients started on fibrates between 2002 and 2008 in the lipid clinic at Good Hope Hospital (part of the Heart of England NHS Foundation Trust) were identified from the electronic patient record database by using appropriate search keywords. Data was collected from 132 consecutive case notes of patients. Lifestyle advice was given to all patients at the initial appointment and this preceded fibrate treatment. The pre-fibrate treatment TC, TG, HDL-C, calculated LDL$\mathrm{C}$ and creatinine concentrations were obtained from the pathology database just prior to fibrate initiation. The post- 


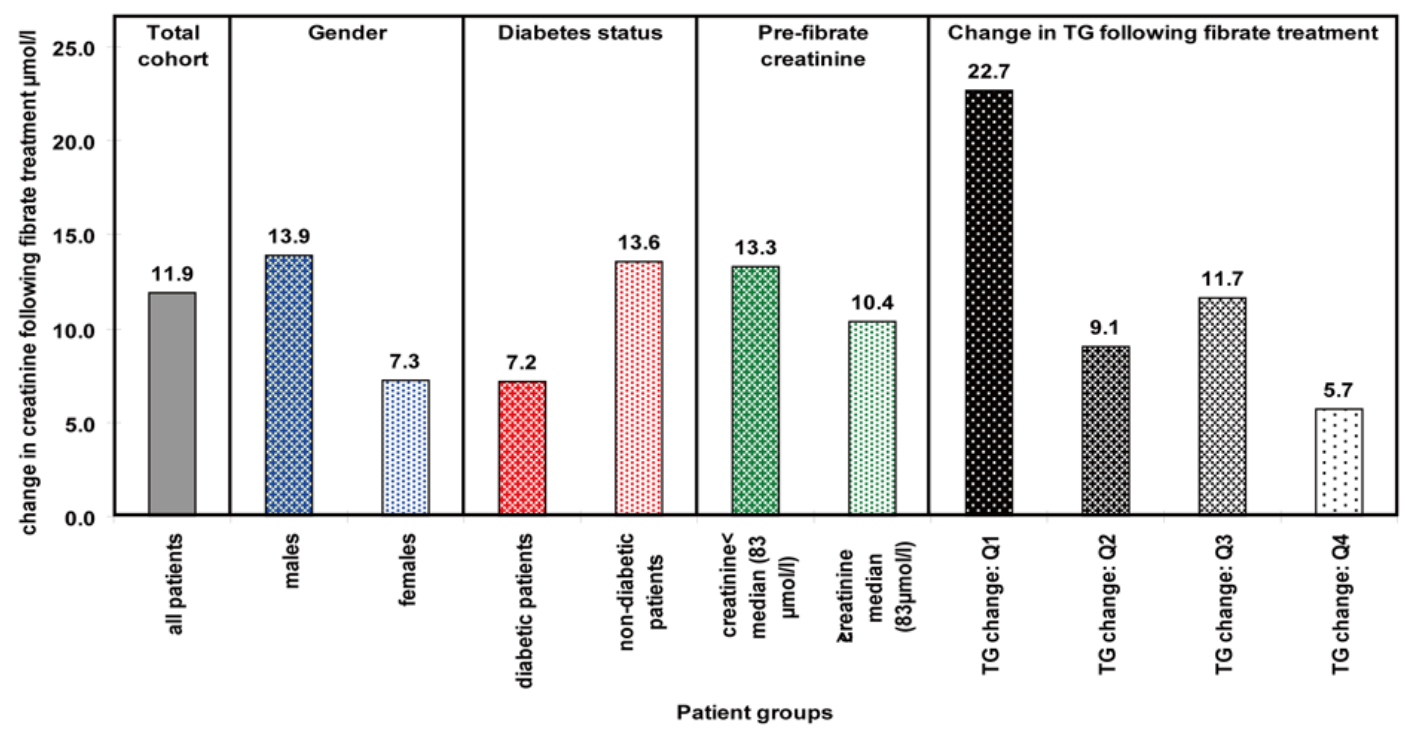

Figure 1. Mean change in creatinine seen within significantly associated factors.

treatment levels were the most recent results available (up to 30.03.2009) or prior to addition of another agent that could affect lipid values or glycaemic control.

TC, TG, HDL-C and creatinine (Jaffe method) levels were measured on the Roche Modular platform P800 analyser using Roche reagents. According to the manufacturers data no significant interference in creatinine analysis was observed up to a lipaemic index of 1000; creatinine values not reported when lipaemic index exceeded this figure. TG content did not appear to correlate with the lipaemic index.

The data was entered on an excel spreadsheet and then transferred to the STATA (version 8.0 for Windows) statistics programme for analysis. Paired $t$ test was performed to determine significant change in creatinine following fibrate treatment. Linear and multiple regression analyses were carried out to study factors that were associated with change in serum creatinine. When the independent variable was not continuous, one characteristic of the variable was chosen as the reference category and the other characteristics of that variable were factorised and compared to the reference category with regard to the selected outcome (dependent variable).

\section{Results}

Fenofibrate was used in $117(88.6 \%)$ patients while the remaining 15 patients were on bezafibrate. Males made up $69.7 \%$ of the cohort (92 patients). It was observed that males had a significantly higher $(\mathrm{P}=0.0001$ on $\mathrm{t}$ test $)$ baseline creatinine concentration $($ mean $=86.0 \mu \mathrm{mol} / \mathrm{L})$ compared to females $($ mean $=73.4 \mu \mathrm{mol} / \mathrm{L})$.

Creatinine increased significantly (paired t test, $\mathrm{P}<$
$0.001)$ from a mean of $81.9(\mathrm{sd}=17.3$, median $=83$, range: $33-127) \mu \mathrm{mol} / \mathrm{L}$ to $93.8(\mathrm{sd}=20.4$, median $=91$, range: 52 - 143) $\mu \mathrm{mol} / \mathrm{L}$ following fibrate treatment. Table 1 demonstrates that a significant increase in creatinine was observed in all patient subgroups (regardless of gender, diabetes status and concurrent statin treatment).

\section{Association between baseline characteristics and change in creatinine levels}

We investigated pre-treatment factors that were associated with the change in creatinine, by carrying out separate linear regression analyses with creatinine change (dependent variable) and baseline factors as well as duration of treatment( independent variables); Table 2- model 1. Male gender (coefficient $=+6.64$, reference: female gender) and lower baseline creatinine levels (coefficient $=-0.20$ ) were significantly associated with greater creatinine increase compared to females and higher baseline creatinine levels respectively. A smaller rise in creatinine levels was observed in patients with diabetes (coefficient $=-6.37$ ) compared to non diabetic patients.

Combination of gender and baseline creatinine in relation to changes in creatinine following fibrate treatment

We have seen that males had a significantly higher pretreatment creatinine level. As male gender and lower pretreatment creatinine levels were associated with greater increase in creatinine following fibrate treatment we wished to further study the relationship between these two factors. Four new combinations based on creatinine levels (above or below median level: $83 \mu \mathrm{mol} / \mathrm{L}$ ) and gender were created 
and entered as factorised independent variables in a linear regression. The reference category chosen in this analysis consisted of female patients with baseline creatinine below the median as this was the subgroup containing the largest number of patients. It was observed that male patients with a pre-treatment creatinine below the median were the only group significantly associated with the increase in creatinine (coefficient $=11.37,95 \% \mathrm{CI}+3.98 /+18.76, \mathrm{P}=0.003) . \mathrm{We}$ then compared this group with the remaining patient subgroups (as reference) and it remained significant (coefficient $=9.41,95 \% \mathrm{CI}+3.73 /+15.08, \mathrm{P}=0.001)$.

\section{Association between lipid lowering and change in creati- nine levels}

We wished to study whether fibrate efficacy with regards lipid lowering was related to the increase in creatinine observed. Baseline TC (mean/median 6.7/6.1 mmol/L), HDL$\mathrm{C}$ (mean/median 1.1/1.1 mmol/L) and TG levels (mean/median $7.4 / 5.3 \mathrm{mmol} / \mathrm{L}$ ) were not associated with the creatinine change following fibrate introduction; the multiple regression model was corrected for length of treatment as we have seen that this was associated with changes in lipid values (doi:10.1089/met.2011.0112). We then wished to determine if the change in creatinine was associated with changes in lipid values. In our patients there were significant changes in lipid levels following fibrate treatment (mean/median changes: HDL-C increase 0.059/0.10 $\mathrm{mmol} / \mathrm{L}, \mathrm{TC}$ decrease $0.91 / 0.50 \mathrm{mmol} / \mathrm{L}$, TG decrease 3.61/2.35 $\mathrm{mmol} / \mathrm{L}$ ). The changes observed in TC (coefficient $=-2.52,95 \% \mathrm{CI}$ : $-3.62 /-1.43, \mathrm{P} \leq 0.001$ ) and TG (coefficient $=-1.02,95 \%$ CI: $-1.39 /-0.65, \mathrm{P} \leq 0.001)$ were associated with the change seen in creatinine. No such significant association was seen with HDL-C (coefficient $=+5.75,95 \%$ CI: $-6.69 /+18.20, \mathrm{P}$ $=0.36)$. As co-linearity was observed we entered TC, TG and HDL-C changes following fibrate treatment into a multiple regression model (Table 2, model 2) with changes observed in creatinine as the dependent variable and only the TG change remained significantly associated with change in creatinine.

We wished to further study the nature of this association and categorised the change in TG levels into quartiles as follows. Q1: TG decrease $>4.3 \mathrm{mmol} / \mathrm{L}, \mathrm{Q} 2$ : TG decrease between $2.51 \mathrm{mmol} / \mathrm{L}$ and $4.3 \mathrm{mmol} / \mathrm{L}, \mathrm{Q} 3$ : TG decrease between $0.81 \mathrm{mmol} / \mathrm{L}$ and $2.50 \mathrm{mmol} / \mathrm{L}, \mathrm{Q} 4:$ TG decrease < $0.80 \mathrm{mmol} / \mathrm{L}$ up to an increase of $5.2 \mathrm{mmol} / \mathrm{L}$. Further regression analysis was carried out with the above factorised quartiles as independent variables (Q4 = reference category) and creatinine change as the dependent variable, with the analysis corrected for duration of fibrate treatment. It was observed that only the quartile with the largest decrease in TG (Q1) was significantly associated with an increase in creatinine (coefficient $=+15.47,95 \%$ CI $+8.14 /+22.80$, $\mathrm{P} \leq 0.001)$. Q1 remained significantly associated with the increase in creatinine when compared to Q2 (coefficient $=$ $+12.74,95 \% \mathrm{CI}:+5.44 /+20.03, \mathrm{P}=0.001)$ and Q3 (coefficient $=+10.87,95 \% \mathrm{CI}:+3.52 /+18.22, \mathrm{P}=0.004)$.

\section{Change in creatinine observed in all the risk factors iden- tified by our study}

We now carried out a similar multiple regression analysis with all the significant factors observed previously as independent variables. Male gender, diabetes, pre-treatment creatinine and decrease in TG levels all remained significant. The mean increase in creatinine levels seen in these patients is presented in Figure 1. We would have liked to have studied interactions between all the above groups as this could have potentially led to useful pointers regards mechanism for the increase in creatinine. However, our patient numbers restricted us from this.

\section{Discussion}

A reversible increase in creatinine has been reported previously in patients, both with normal baseline creatinine and impaired renal function, treated with fenofibrate, bezafibrate and ciprofibrate $[8,10,12,13,15-18]$. Gemfibrozil in some surveys appears less associated with this phenomenon [15]. Interestingly, clinical trials with the dual PPAR $\alpha / \gamma$ agonist tesaglitazar demonstrated an elevation in serum creatinine $[19,20]$. Although well recognised, the mechanisms of this phenomenon and the patient groups at risk remain unclear. Our retrospective study of patients treated with fibrates in an outpatient clinic revealed the mean creatinine levels to increase significantly by $11.9 \mu \mathrm{mol} / \mathrm{L}$. This increase was significantly associated with male gender, lower pre-treatment creatinine, non-diabetic patients and patients demonstrating the greatest reduction in plasma TG values. None of the above factors have previously been associated with the increase in creatinine. In contrast to our findings, Broeders et al on studying the effects of gemfibrozil concluded that hypercreatininaemia was not associated with age, gender or pre-treatment creatinine levels [15]. As gemfibrozil is less associated with this phenomenon one can speculate the possibility of within class variation.

PPAR $\alpha$ is expressed in tissues demonstrating higher rates of fatty acid catabolism such as the kidneys, skeletal muscle, cardiac muscle and liver [21]. Fibrates bind to the PPAR $\alpha$-RXR complex, which is involved in the regulation of a number of genes coding for proteins involved in lipoprotein metabolism with $\mathrm{TG}$ reduction one of its principal effects [1]. It has been reported that the change in lipid values is related to the nature of the binding between fibrates and PPAR $\alpha$-RXR [22]. We have observed that the creatinine increase following fibrate treatment was associated with the change in TG levels. Thus, we can speculate that the nature 
of the binding of fibrates to PPAR $\alpha-R X R$, either in the kidneys or muscle may play some part in this phenomenon.

It has been suggested that the increased creatinine may signify genuine renal impairment due to PPAR $\alpha$ mediated down-regulation of cyclooxygenase resulting in reduced vasodilatory prostaglandins in the kidney [15]. Studies supporting this hypothesis provide evidence that gemfibrozil, the fibrate least associated with hypercreatininaemia, does not inhibit the production of vasodilatory prostaglandins as opposed to other fibrates such as clofibrate or ciprofibrate $[23,24]$. None of our patients were on gemfibrozil. Creatinine levels are dependent on both glomerular filtration and tubular secretion in the kidney via an organic cation receptor in the proximal tubule [25]. There is no evidence that fibrates (and their metabolites) which are organic anions affect renal tubular secretion of creatinine [26]. In fact a rise in urinary creatinine levels have been observed in patients experiencing hypercreatininaemia following fibrate therapy [9, 10]. Several studies which have measured GFR in patients with creatinine elevation (up to a $20 \%$ increase) following fibrate treatment have not demonstrated glomerular impairment using inulin [8-10]. Significant direct nephrotoxicity induced by fibrates within the commonly observed range of creatinine increase is unlikely as no increase in proteinuria has previously been reported with these drugs; in fact a reduction in the rate of onset and progression of microalbuminuria has been described [27]. A recent animal study by Ovcharenko et al. did not reveal any evidence of a direct nephrotoxic effect or deterioration in renal haemodynamics caused by either PPAR $\alpha$ (fenofibrate) or dual PPAR $\alpha / \gamma$ (tesaglitazar) agonists [28].

Another view is that increased creatinine production from muscle is the most likely cause of hypercreatininaemia following fibrate use [26]. PPAR $\alpha$ is a regulator of fatty acid oxidation in skeletal muscle [29]. Measurement of mRNA in skeletal muscle biopsies demonstrated that mRNA of PPAR $\alpha$ was correlated with the mRNA of lipoprotein lipase and muscle CPT2, both enzymes involved with fatty acid metabolism [30]. There has also been a suggestion that part of the lipid lowering effects of PPAR activators may be mediated by muscle clearance and utilisation of plasma lipids [31]. However, no reports exist of a correlation between fatty acid oxidation in the muscle and hypercreatininaemia. Interestingly muscle damage has been reported in patients with the myopathic form of CPT2 deficiency, perhaps due to impaired ability to utilise fatty acids [32]. This again does not provide an explanation to the association between TG change and hypercreatininaemia.

We have observed that the increase in creatinine following fibrates was associated with male gender and lower baseline creatinine levels. Males have a significantly higher creatinine than females due to a greater muscle mass [33] and this was also the case in our study $(\mathrm{P}<0.0001$, unpaired t-test). Not surprisingly, both these factors were independent of each other. We could not study the interaction between gender and baseline creatinine and the effect on the change in creatinine in great detail due to limited patient numbers. Interestingly, studies have demonstrated that oestrogen has an inhibitory effect on the actions of PPAR $\alpha$ on lipid metabolism [34]. Gender differences have also been seen with PPAR $\alpha$ expression greater in T cells of male mice; this expression being sensitive to androgen levels [35]. It would be interesting to study androgen and oestrogen levels amongst our patient cohort and investigate any correlation with change in creatinine. We also noted that creatinine increases were greater in non-diabetic patients. There has been no observed difference in a functional PPAR $\alpha$ polymorphism (Leu162Val) between diabetic and non-diabetic patients [36]. Thus, once again we cannot provide any plausible explanations for these observations.

We have presented data from a retrospective study examining the use of fibrates in an outpatient setting. There are limitations to this kind of work. However, they are often useful in providing initial observations that must be validated by a more controlled study. The increase in creatinine following fibrates was similar to that observed in randomized controlled trials such as FIELD. We speculated that it may be related to PPAR $\alpha$ activity in view of the association with TG reduction. However, current knowledge of PPAR $\alpha$ receptors does not allow us to speculate any further. We acknowledge that regression to the mean with regards our findings should be considered. It is essential that prospective confirmatory studies be designed to confirm patient phenotypes and mechanisms that are associated with creatinine change associated with fibrates.

\section{Abbreviations}

TG: Triglycerides; PPAR $\alpha$ : Peroxisome Proliferator-Activated Receptor $\alpha$; LDL-C: Low Density Lipoprotein Cholesterol; HDL-C: High Density Lipoprotein Cholesterol; FIELD: Fenofibrate Intervention and Event Lowering In Diabetes; HPLC: High Performance Liquid Chromatography; GFR: Glomerular Filtration Rate; NKD: National Kidney Foundation; NLA: National Lipid Association; TC: Total Cholesterol; RXR: Retinoid X Receptor; mRNA: Messenger Ribonucleic Acid; CPT2: Carnitine Palmitoyltransferase 2.

\section{References}

1. Staels B, Dallongeville J, Auwerx J, Schoonjans K, Leitersdorf E, Fruchart JC. Mechanism of action of fibrates on lipid and lipoprotein metabolism. Circulation. 1998;98(19):2088-2093.

2. Chapman MJ. Fibrates in 2003: therapeutic action in atherogenic dyslipidaemia and future perspectives. Ath- 
erosclerosis. 2003;171(1):1-13.

3. Fazio S, Linton MF. The role of fibrates in managing hyperlipidemia: mechanisms of action and clinical efficacy. Curr Atheroscler Rep. 2004;6(2):148-157.

4. Farnier M. Update on the clinical utility of fenofibrate in mixed dyslipidemias: mechanisms of action and rational prescribing. Vasc Health Risk Manag. 2008;4(5):9911000.

5. Levey AS, Perrone RD, Madias NE. Serum creatinine and renal function. Annu Rev Med. 1988;39:465-490.

6. Ginsberg HN, Elam MB, Lovato LC, Crouse JR, 3rd, Leiter LA, Linz P, Friedewald WT, et al. Effects of combination lipid therapy in type 2 diabetes mellitus. N Engl J Med. 2010;362(17):1563-1574.

7. Forsblom C, Hiukka A, Leinonen ES, Sundvall J, Groop PH, Taskinen MR. Effects of long-term fenofibrate treatment on markers of renal function in type 2 diabetes: the FIELD Helsinki substudy. Diabetes Care. 2010;33(2):215-220.

8. Hottelart C, el Esper N, Achard JM, Pruna A, Fournier A. [Fenofibrate increases blood creatinine, but does not change the glomerular filtration rate in patients with mild renal insufficiency]. Nephrologie. 1999;20(1):4144.

9. Ansquer JC, Dalton RN, Causse E, Crimet D, Le Malicot $\mathrm{K}$, Foucher C. Effect of fenofibrate on kidney function: a 6-week randomized crossover trial in healthy people. Am J Kidney Dis. 2008;51(6):904-913.

10. Hottelart C, El Esper N, Rose F, Achard JM, Fournier A. Fenofibrate increases creatininemia by increasing metabolic production of creatinine. Nephron. 2002;92(3):536-541.

11. Davidson MH, Armani A, McKenney JM, Jacobson TA. Safety considerations with fibrate therapy. Am J Cardiol. 2007;99(6A):3C-18C.

12. Lipscombe J, Lewis GF, Cattran D, Bargman JM. Deterioration in renal function associated with fibrate therapy. Clin Nephrol. 2001;55(1):39-44.

13. Tsimihodimos V, Kakafika A, Elisaf M. Fibrate treatment can increase serum creatinine levels. Nephrol Dial Transplant. 2001;16(6):1301.

14. K/DOQI clinical practice guidelines for chronic kidney disease: evaluation, classification, and stratification. Am J Kidney Dis. 2002;39(2 Suppl 1):S1-266.

15. Broeders N, Knoop C, Antoine M, Tielemans C, Abramowicz D. Fibrate-induced increase in blood urea and creatinine: is gemfibrozil the only innocuous agent? Nephrol Dial Transplant. 2000;15(12):1993-1999.

16. Ritter JL, Nabulsi S. Fenofibrate-Induced elevation in serum creatinine. Pharmacotherapy. 2001;21(9):11451149.

17. Rizos E, Bairaktari E, Ganotakis E, Tsimihodimos V, Mikhailidis DP, Elisaf M. Effect of ciprofibrate on lipoproteins, fibrinogen, renal function, and hepatic en- zymes. J Cardiovasc Pharmacol Ther. 2002;7(4):219226.

18. Charach G, Grosskopf I, Rotmensch HH, Kitzis I, Weintraub M. Bezafibrates cause moderate, reversible impairment in renal function in patients without prior renal disease. Nephron Clin Pract. 2005;100(4):c120-125.

19. Bays H, McElhattan J, Bryzinski BS. A double-blind, randomised trial of tesaglitazar versus pioglitazone in patients with type 2 diabetes mellitus. Diab Vasc Dis Res. 2007;4(3):181-193.

20. Ratner RE, Parikh S, Tou C. Efficacy, safety and tolerability of tesaglitazar when added to the therapeutic regimen of poorly controlled insulin-treated patients with type 2 diabetes. Diab Vasc Dis Res. 2007;4(3):214-221.

21. Lemberger T, Braissant O, Juge-Aubry C, Keller H, Saladin R, Staels B, Auwerx J, et al. PPAR tissue distribution and interactions with other hormone-signaling pathways. Ann N Y Acad Sci. 1996;804:231-251.

22. Panadero MI, Gonzalez MC, Herrera E, Bocos C. Factors modulating fibrates response: therapeutic implications and alternative strategies. Endocr Metab Immune Disord Drug Targets. 2009;9(3):219-236.

23. Ledwith BJ, Pauley CJ, Wagner LK, Rokos CL, Alberts DW, Manam S. Induction of cyclooxygenase-2 expression by peroxisome proliferators and non-tetradecanoylphorbol 12,13-myristate-type tumor promoters in immortalized mouse liver cells. J Biol Chem. 1997;272(6):3707-3714.

24. Yoshinari M, Asano T, Kaori S, Shi AH, Wakisaka M, Iwase M, Fujishima M. Effect of gemfibrozil on serum levels of prostacyclin and precursor fatty acids in hyperlipidemic patients with Type 2 diabetes. Diabetes Res Clin Pract. 1998;42(3):149-154.

25. Urakami Y, Kimura N, Okuda M, Inui K. Creatinine transport by basolateral organic cation transporter hOCT2 in the human kidney. Pharm Res. 2004;21(6):976-981.

26. Sica DA. Fibrate therapy and renal function. Curr Atheroscler Rep. 2009;11(5):338-342.

27. Ansquer JC, Foucher C, Rattier S, Taskinen MR, Steiner G. Fenofibrate reduces progression to microalbuminuria over 3 years in a placebo-controlled study in type 2 diabetes: results from the Diabetes Atherosclerosis Intervention Study (DAIS). Am J Kidney Dis. 2005;45(3):485493.

28. Ovcharenko E, Hansen MK, Goltsman I, Abu-Saleh N, Abassi Z, Walsh K, Miele G, et al. Peroxisome proliferator-activated receptor alpha and alpha/gamma agonists do not cause impairment in renal function in the rat. Nephron Physiol. 2010;115(3):p21-30.

29. Muoio DM, Way JM, Tanner CJ, Winegar DA, Kliewer SA, Houmard JA, Kraus WE, et al. Peroxisome proliferator-activated receptor-alpha regulates fatty acid utilization in primary human skeletal muscle cells. Diabetes. 2002;51(4):901-909. 
30. Zhang J, Phillips DI, Wang C, Byrne CD. Human skeletal muscle PPARalpha expression correlates with fat metabolism gene expression but not BMI or insulin sensitivity. Am J Physiol Endocrinol Metab. 2004;286(2):E168175.

31. Srivastava RA, Jahagirdar R, Azhar S, Sharma S, Bisgaier CL. Peroxisome proliferator-activated receptor-alpha selective ligand reduces adiposity, improves insulin sensitivity and inhibits atherosclerosis in LDL receptordeficient mice. Mol Cell Biochem. 2006;285(1-2):35-50.

32. Orngreen MC, Duno M, Ejstrup R, Christensen E, Schwartz M, Sacchetti M, Vissing J. Fuel utilization in subjects with carnitine palmitoyltransferase 2 gene mutations. Ann Neurol. 2005;57(1):60-66.

33. Toto RD. Conventional measurement of renal function utilizing serum creatinine, creatinine clearance, inulin and para-aminohippuric acid clearance. Curr Opin
Nephrol Hypertens. 1995;4(6):505-509; discussion 503504.

34. Yoon M. The role of PPARalpha in lipid metabolism and obesity: focusing on the effects of estrogen on PPARalpha actions. Pharmacol Res. 2009;60(3):151-159.

35. Dunn SE, Ousman SS, Sobel RA, Zuniga L, Baranzini SE, Youssef S, Crowell A, et al. Peroxisome proliferator-activated receptor (PPAR)alpha expression in T cells mediates gender differences in development of $\mathrm{T}$ cellmediated autoimmunity. J Exp Med. 2007;204(2):321330.

36. Sparso T, Hussain MS, Andersen G, Hainerova I, BorchJohnsen K, Jorgensen T, Hansen T, et al. Relationships between the functional PPARalpha Leu162Val polymorphism and obesity, type 2 diabetes, dyslipidaemia, and related quantitative traits in studies of 5799 middle-aged white people. Mol Genet Metab. 2007;90(2):205-209. 